\title{
Consequences of Biomarker Analysis on the Cost-Effectiveness of Cetuximab in Combination with FOLFIRI as a First-Line Treatment of Metastatic Colorectal Cancer: Personalised Medicine at Work
}

\author{
Gerard Harty $^{1} \cdot$ James Jarrett $^{2} \cdot$ Mireia Jofre-Bonet $^{3}$
}

Published online: 9 June 2018

(C) The Author(s) 2018

\begin{abstract}
Background Therapies may be more efficacious when targeting a patient subpopulation with specific attributes, thereby enhancing the cost-effectiveness of treatment. In the CRYSTAL study, patients with metastatic colorectal cancer (mCRC) were treated with cetuximab plus FOLFIRI or FOLFIRI alone until disease progression, unacceptable toxic effects or withdrawal of consent.

Objective To determine if stratified use of cetuximab based on genetic biomarker detection improves costeffectiveness.

Methods We used individual patient data from CRYSTAL to compare the cost-effectiveness, cost per life-year (LY) and cost per quality-adjusted LY (QALY) gained of cetuximab plus FOLFIRI versus FOLFIRI alone in three cohorts of patients with mCRC: all randomised patients (intent-to-treat; ITT), tumours with no detectable mutations in codons 12 and 13 of exon 2 of the KRAS protein ('KRAS wt') and no detectable mutations in exons 2, 3 and 4 of KRAS and exons 2, 3 and 4 of NRAS ('RAS wt'). Survival analysis was conducted using RStudio, and a costutility model was modified to allow comparison of the three cohorts.
\end{abstract}

Electronic supplementary material The online version of this article (https://doi.org/10.1007/s40258-018-0395-5) contains supplementary material, which is available to authorised users.

Mireia Jofre-Bonet

mireia.jofre-bonet.1@ city.ac.uk

1 Merck Serono Ltd, Bedfont Cross, Stanwell Road, Feltham, Middlesex TW14 8NX, UK

2 Mapi Group, Ltd, 73 Collier Street, London N1 9BE, UK

3 City University London, Northampton Square, London EC1V 0HB, UK
Results The deterministic base-case ICER (cost per QALY gained) was $£ 130,929$ in the ITT, $£ 72,053$ in the KRAS wt and $£ 44,185$ in the RAS wt cohorts for cetuximab plus FOLFIRI compared with FOLFIRI alone. At a $£ 50,000$ willingness-to-pay threshold, cetuximab plus FOLFIRI has a $2.8,20$ and $63 \%$ probability of being cost-effective for the ITT, KRAS wt and RAS wt cohorts, respectively, versus FOLFIRI alone.

Conclusion Screening for mutations in both KRAS and NRAS may provide the most cost-effective approach to patient selection.

\section{Key Points for Decision Makers}

Adding cetuximab to FOLFIRI is more cost-effective in patients with metastatic colorectal cancer (mCRC) and a wild-type RAS gene than in the mCRC population at large.

At a $£ 50,000$ willingness-to-pay threshold, cetuximab plus FOLFIRI has $63 \%$ probability of being cost-effective among patients with the wildtype RAS gene compared with a $2.8 \%$ probability among the mCRC population at large.

These results demonstrate potential economic benefits of personalised medicine based on biomarker testing. 


\section{Introduction}

Personalised medicine uses information about specific patient biological attributes and environment to most effectively prevent, diagnose or treat disease. Biomarker testing for a particular biological attribute is one way through which patients may be differentiated [1-4]. The premise underlying personalised medicine is that therapies demonstrating a particular therapeutic outcome for an overall disease population may show greater effect when targeting a subgroup with a certain set of attributes. Aside from enabling practitioners to more effectively treat patients, personalised medicine may also benefit healthcare systems through enhancing the cost-effectiveness of a particular treatment [5]. In oncology, biomarker testing and stratified medicine may allow development of precision care plans using the most appropriate medication given the biological status of a patient's tumour [6]. Improved outcomes translate directly into improved cost-effectiveness if the benefit to the patient outweighs the increased costs to the healthcare system, such as from additional drug acquisition and administration and biomarker testing costs [7].

Colorectal cancer (CRC) is one of the most common cancers in the United Kingdom (UK), with an annual incidence of about 40,000 patients [8]. CRC is one of several cancer types associated with overexpression of the epidermal growth factor receptor (EGFR) signalling pathway. Signalling through this pathway results in cell proliferation, inhibition of apoptosis, activation of invasion, metastasis and angiogenesis [9-11]. Based on the role of EGFR in cancer, anti-EGFR therapy has been introduced as an approach to reduce intracellular signalling. There are several molecular components downstream of the EGFR pathway that help regulate the effects of EGFR stimulation. The rat sarcoma oncogene (RAS) proteins Kirsten rat sarcoma viral oncogene homolog (KRAS) and neuroblastoma RAS viral oncogene homolog (NRAS) are components of the second-messenger signalling pathway initiated by EGFR, and they help regulate the cell cycle [9-12]. In some patients, RAS proteins harbour mutations that render these proteins unaffected by any changes induced by antiEGFR-based treatment $[13,14]$. Patients with these mutations can be identified by biomarker testing $[12,15,16]$.

Cetuximab is an immunoglobulin G1 monoclonal antibody that targets EGFR, and has been licensed since 2008 in combination with 5-fluorouracil and leucovorin plus oxaliplatin (FOLFOX) and with 5-fluorouracil and leucovorin plus irinotecan (FOLFIRI) chemotherapy for firstline use in patients with metastatic CRC (mCRC). The pivotal CRYSTAL study was an open-label, randomised, controlled, multicentre phase 3 trial that compared
FOLFIRI plus cetuximab versus FOLFIRI alone as firstline therapy for EGFR-expressing mCRC [14, 17, 18]. Study results showed significantly longer progression-free survival (PFS) with cetuximab plus FOLFIRI compared with FOLFIRI alone [17]. In the intent-to-treat (ITT) population, comprising all patients randomised to receive treatment, the cetuximab-plus-FOLFIRI arm showed a median overall survival (OS) of 19.9 months compared with 18.6 months in the FOLFIRI-alone arm, corresponding to a 1.3-month benefit with a hazard ratio (HR) of 0.93 (95\% CI $0.81-1.07 ; P=0.31$ ) [17]. Based on the role of the EGFR pathway in mCRC, investigators examined subpopulations of patients defined by RAS genotype; specifically, KRAS wild-type (wt) patients (tumours had no detectable mutations in codons 12 and 13 of exon 2 of KRAS), and RAS wt patients [meeting KRAS wt criteria plus no detectable mutations in exons 2 (codons 12 and 13) of NRAS, 3 (codons 59 and 61) and 4 (codons 117 and 146) of KRAS and NRAS] [14].

When comparing median OS in the cetuximab-plusFOLFIRI arm versus the FOLFIRI-alone arm in biomarkerselected subgroups, patients with a KRAS wt genotype demonstrated a 3.5 -month benefit in median OS [23.5 vs. 20.0 months, respectively; HR, 0.796 (95\% CI 0.670-0.946); $P=0.0093$ ] [18], while patients in the RAS wt subgroup showed an 8.2-month benefit in median OS [28.4 vs. 20.2 months, respectively; HR, 0.69 (95\% CI 0.54-0.88); $P=0.0024$ ] [14]. On the basis of these and other data demonstrating enhanced efficacy of EGFR inhibitors in patients with RAS wt tumours, clinical practise guidelines recommend testing for RAS status prior to determining first-line treatment for patients with mCRC, and the indication for cetuximab was updated accordingly $[19,20]$.

Economic analyses of the cost-effectiveness of cetuximab have been submitted to several UK regulatory bodies for health technology assessments (HTAs), resulting in recommendations for restricted use (Table 1). In the current study, an objective economic evaluation was performed using CRYSTAL trial cohorts to determine if stratified use of cetuximab based on genetic biomarker detection improves cost-effectiveness. This investigation was based on the hypothesis that treating the population(s) that benefit(s) the most should improve the costeffectiveness of the intervention. This is the first time a common model or platform has been used so that the incremental cost-effectiveness ratios (ICERs) between three populations can be examined objectively in patients with mCRC. 
Table 1 Previous cetuximab submissions for UK health technology assessment

\begin{tabular}{|c|c|c|c|c|}
\hline Date/reference & HTA group & HTA ID & Population & Outcome \\
\hline 04 Apr 2009 [21] & SMC & $543 / 09$ & First-line in all patients with KRAS wt mCRC & Not recommended \\
\hline 01 Aug 2009 [22] & NICE & TA176 & $\begin{array}{l}\text { First-line in subgroup of patients with } \\
\text { liver-limited KRAS wt mCRC }\end{array}$ & Recommended for restricted use \\
\hline 08 Feb 2010 [21] & SMC & 543/09/Resubmission & $\begin{array}{l}\text { First-line in subgroup of patients with } \\
\text { liver-limited KRAS wt mCRC }\end{array}$ & Recommended for restricted use \\
\hline 12 Jan 2015 [23] & SMC & $1012 / 14$ & First-line in all patients with RAS wt mCRC & Recommended for restricted use \\
\hline 24 Feb 2016 [24] & AWMSG & 4315 & First-line in patients with RAS wt mCRC & Recommended for restricted use \\
\hline March 2017 [25] & NICE & Pending & $\begin{array}{l}\text { First-line in RAS wt mCRC in combination } \\
\text { with FOLFOX or FOLFIRI }\end{array}$ & Recommended \\
\hline
\end{tabular}

AWMSG All Wales Medicines Strategy Group, FOLFIRI 5-fluorouracil and leucovorin plus irinotecan, FOLFOX 5-fluorouracil and leucovorin plus oxaliplatin, HTA health technology assessment, ID identification, KRAS Kirsten rat sarcoma viral oncogene homolog, $m C R C$ metastatic colorectal cancer, NICE National Institute for Health and Care Excellence, RAS rat sarcoma oncogene, SMC Scottish Medicines Consortium, wt wild type

\section{Methods}

Individual patient data (IPD) from the CRYSTAL study were obtained from Merck KGaA (Darmstadt, Germany). All patients in the CRYSTAL study provided written and oral informed consent [17]. Results were categorised into three cohorts: the ITT population, the KRAS wt subgroup and the RAS wt subgroup (defined above) [14], comparing cetuximab plus FOLFIRI versus FOLFIRI alone. FOLFOX was not investigated, because the phase 2 clinical trial that compared the use of cetuximab in combination with FOLFOX versus FOLFOX alone (OPUS) had a smaller patient population, and, thus, greater volatility around the results [26]. Tumour genotypes were determined using a polymerase-chain-reaction technique on DNA extracted from tumour-biopsy specimens. Screening for KRAS mutations in codons 12 and 13 was performed initially and then samples determined as KRAS wt were subsequently screened for the RAS genotype [14, 17].

For the cost-effectiveness comparison, a proprietary, validated cost-utility model was used to compare cetuximab-based first-line treatment regimens with irinotecanbased chemotherapy for patients with mCRC based on PFS, adverse events (AEs) and resource usage. Utilities were obtained from quality-of-life (QOL) data collected during the original CRYSTAL trial [27]. The model was created using a UK National Health Service (NHS) perspective; therefore, only direct costs to the NHS were included. The model was developed in Microsoft Excel with Visual Basic codes to conduct one-way sensitivity analyses (OWSA) and probabilistic sensitivity analyses (PSA) for appropriate pairwise comparisons [28]. The core of the model is a state-transition Markov cohort method developed to simulate patient outcomes and costs for first and subsequent lines of oncology treatment, including long-term survival after a successful curative resection of liver metastases. As opposed to area-under-the-curve analyses, this model is a Markov state and transition model with the probabilities of transitions dependent on time from the beginning of treatment of cohort and on time in state. This means the probability of transition from second-line to third-line therapy is dependent on the time of progression from first-line therapy to second-line therapy. To apply these time-dependent transition probabilities for this sequence of treatments, the model uses tunnel states. This model structure is based on previous models submitted to HTA bodies and subsequently published [29, 30]. To provide a distribution around each parameter, an upper and lower limit was generated by using the 0.025 percentile as a lower bound and the 0.975 percentile as an upper bound of the relevant variable. The specific model used was based on an earlier model developed to demonstrate the costeffectiveness in the RAS wt cohort alone (see Ref. [29]). The current model was modified so that all three cohorts (ITT, KRAS wt and RAS wt) within the CRYSTAL study could be analysed together. The main adaptive changes within the current model affect a patient's progression through the first-line heath state, the curative resection rates and the frequency of AEs between cohorts.

A disease-modelling approach (assuming the PFS benefits of first-line cancer treatments translate directly into OS benefits) was selected to examine costs and benefits outside the trial period to compare cohorts over a longer time horizon than the study provided. This model was previously assessed by a National Institute for Health and Care Excellence (NICE) Evidence Review Group (ERG) [22]. The time horizon was set to 10 years, which was considered reasonable to capture all costs and benefits, including those of patients who receive curative surgery, and is consistent with previous HTA submissions (Table 1). The NICE discounting policy was followed, which is to apply a $3.5 \%$ discount rate for costs and 
benefits. We also made no distinction between no resection and unsuccessful resection, because it may only become apparent after initiation of treatment if surgery is an option [31]. The model was updated by constructing the time-toevent data from the IPD for the ITT, KRAS wt and RAS wt cohorts. Model inputs are provided in Table S1. These parameters were then used to generate the transition probability for the Markov cycles. Adverse events (Table S2) and resection rates (Table S3) were also collated. The risk of progression from first-line treatment was applied in the model using the Weibull parametric extrapolations of the time to progression. $\mathrm{R}$ and RStudio (version 3.1.2) were used to derive the numerical coefficients for this distribution. The IPD PFS time in the data was converted to weeks to match the transition cycles within the model and uploaded into RStudio. The Weibull parametric model was fitted using 'survreg()' [32], then outputs from RStudio created from the model were entered to determine new extrapolation distributions for each of the three cohorts. The proportional hazards assumption was made for the Kaplan-Meier data for the treatment arms from the CRYSTAL trial. Based on experience that the Weibull model was the best fit for this data, further parametric survival analysis was considered to be unnecessary as the objective was to maintain consistency between the core model parameters to compare like with like.

Patients entering the model started in the first-line therapy health state, then either underwent curative resection of liver metastases and entered the postresection health state or progressed to the second-line treatment health state, then to the third-line health state (Fig. 1). Patients received best supportive care as third-line treatment. In the treatment and control arms, second- and third-line progression was not adjusted within the model. It was assumed that the same principles of further progression in the second line, and survival in the third line, were followed for all patients who progressed at different times from the start of first-line treatment. It was also assumed that the survival probability following curative resection was identical for all treatment arms, and survival estimates of curative surgery were based on those reported by Adam et al. [33]. Grade 3/4 AEs from both arms of the three cohorts were sourced and extracted from the CRYSTAL publications $[14,17,18]$ and clinical study reports and entered into the model. Treatment costs (acquisition and administration) for all the health states were derived from the 2013 British National Formulary and UK National Reference Costs (Table S4). AE costs depended on the assumption that there would be an associated outpatient or inpatient visit, and included cost of drugs and outpatient visits. For the ITT analysis, costs associated with biomarker testing were removed from the model as this population would not have been stratified before treatment, but costs were included within the other cohorts. Sensitivity and specificity analysis of the biomarker testing was not conducted, as all patients were presumed to be correctly stratified in the post hoc analysis because these biomarker techniques have high technical accuracy [34].

Cost-effectiveness was measured in terms of costs per life-year (LY) gained and per quality-adjusted life-year (QALY) gained. Both OWSA and PSA were evaluated using standard methods [35]. The PSA was set at 2000 runs and the distributes for the majority of parameters are presented in Table S5. For the OWSA, where the standard error was missing for some variables, it was assumed to be $10 \%$ of the mean value. This was held consistent between all cohorts. As an alternative to comparing the resulting ICERs between groups, two willingness-to-pay (WTP) thresholds were also considered in the model at $£ 30,000$ and $£ 50,000$ ( $£ 30,000$ based on the upper level of the NICE WTP threshold for the UK, and $£ 50,000$ when end-of-life criteria are considered appropriate for a technology) [36].

\section{Results}

Baseline characteristics were generally consistent between the ITT, RAS wt and KRAS wt populations, as described previously [14, 17, 18]. Results of the cost-effectiveness analysis of the three CRYSTAL cohorts are presented in Table 2. In the deterministic base-case ICER cost per LY gained, results for cetuximab plus FOLFIRI versus FOLFIRI alone were $£ 98,742$ for the ITT, 555,242 for

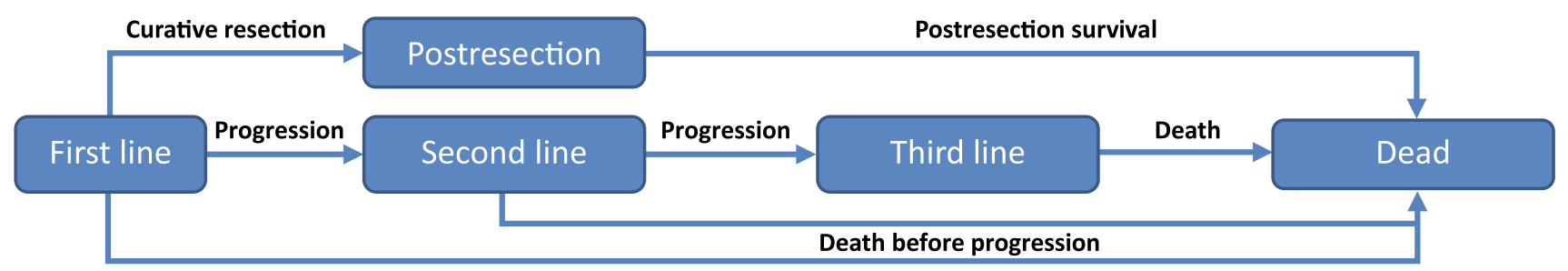

Fig. 1 Demonstration of how patients move between the different health states. Patients entered the model in the first-line therapy health state, then either underwent curative resection of liver metastases and entered the postresection health state or progressed to the second-line treatment health state, then to the third-line health state 
Table 2 Incremental cost-effectiveness results

\begin{tabular}{|c|c|c|c|c|c|}
\hline & Costs $(£)$ & LYs gained & QALYs gained & ICER (cost per LY) & ICER (cost per QALY) \\
\hline \multicolumn{6}{|l|}{ ITT population } \\
\hline Cetuximab + FOLFIRI & 47,643 & 2.05 & 1.50 & & \\
\hline FOLFIRI & 31,840 & 1.89 & 1.38 & & \\
\hline Increment & 15,802 & 0.16 & 0.12 & 98,742 & 130,929 \\
\hline \multicolumn{6}{|l|}{ KRAS wt cohort } \\
\hline Cetuximab + FOLFIRI & 47,712 & 2.29 & 1.69 & & \\
\hline FOLFIRI & 31,840 & 2.00 & 1.47 & & \\
\hline Increment & 15,907 & 0.29 & 0.22 & 55,242 & 72,053 \\
\hline \multicolumn{6}{|l|}{ RAS wt cohort } \\
\hline Cetuximab + FOLFIRI & 47,168 & 2.54 & 1.89 & & \\
\hline FOLFIRI & 31,673 & 2.09 & 1.54 & & \\
\hline Increment & 15,495 & 0.45 & 0.35 & 34,171 & 44,185 \\
\hline
\end{tabular}

FOLFIRI 5-fluorouracil, leucovorin and irinotecan, ICER incremental cost-effectiveness ratio, ITT intent-to-treat, KRAS Kirsten rat sarcoma viral oncogene homolog, $L Y$ life-year, $Q A L Y$ quality-adjusted life-year, $R A S$ rat sarcoma oncogene, $w t$ wild type

the KRAS wt and $£ 34,171$ for the RAS wt cohorts, respectively. The results for the deterministic base-case ICER cost per QALY gained were $£ 130,929$ for the ITT, $£ 72,053$ for the KRAS wt and $£ 44,185$ for the RAS wt cohorts. The analysis output demonstrated that the cetuximab arm resulted in an improvement of $0.16 \mathrm{LYs}$ and 0.12 QALYs among the ITT cohort compared with 0.29 LYs and 0.22 QALYs for KRAS wt and 0.45 LYs and 0.35 QALYs for RAS wt. The incremental cost of treatment per patient changed slightly in the ITT $(£ 15,802)$, KRAS wt $(£ 15,907)$ and RAS wt $(£ 15,495)$ groups, which was mainly driven by the increasing proportion of patients who received curative surgery and no longer required second- or third-line chemotherapy. Together these data suggest that the RAS wt group was the most cost-effective of the cohorts.

The tornado diagrams in Figure 2 show the results of the OWSA on the deterministic base-case ICER for the cetuximab-plus-FOLFIRI versus FOLFIRI-alone comparisons. In all tornado diagrams, the HR of progression from first-line for cetuximab plus FOLFIRI compared with FOLFIRI and the number of months on treatment had the most impact on the model. Resection rates, body surface area and unit cost of cetuximab also had a substantive effect on the ICER. In the ITT cohort, increasing the HR from the base-case value of 0.80 to the upper bound of 1.196 resulted in an ICER of $£ 878,390$. Reducing this HR to its lower bound (0.539) generated an ICER of $£ 65,734$ (Fig. 2a). Variation in the duration of treatment had a large impact on the ICER. Assuming that the lower bound of this variable (3.51 months) generated an ICER of $£ 78,461$, a treatment regimen lasting 8.03 months resulted in an ICER of $£ 176,003$. Resection rates also had a considerable effect on the ICER: the lower rate (0.039) of the cetuximab-plus-
FOLFIRI ITT cohort increased the ICER to $£ 187,890$, and the higher resection rate $(0.087)$ lowered the ICER to $£ 93,904$. In the KRAS wt group, increasing the HR from a base-case value of 0.645 to the upper bound of 0.936 resulted in an ICER of $£ 177,050$. Reducing the HR to its lower bound (0.444) generated an ICER of $£ 42,153$ (Fig. 2b). Variation in the duration of treatment had a large impact on the ICER in this cohort as well, assuming that the lower bound of this variable (3.51 months) generated an ICER of $£ 42,796$, while a treatment regimen lasting 8.03 months resulted in an ICER of $£ 98,675$. The unit cost of cetuximab and the average body surface area had a slightly greater impact than the resection rates on the deterministic base-case ICER in this group. In the RAS wt group, increasing the HR from the base-case value of 0.564 to the upper bound of 0.782 resulted in an ICER of $£ 83,362$. Reducing this HR to its lower bound (0.407) generated an ICER of $£ 27,893$ (Fig. 2c). As in the ITT and KRAS wt cohorts, variation in the duration of treatment also had a large impact on the ICER among patients in the RAS wt cohort, assuming that the lower bound of this variable (3.51 months) generated an ICER of $£ 25,626$, while a treatment regimen lasting 8.03 months resulted in an ICER of $£ 61,409$. Unit cost of cetuximab and the average body surface area had a greater impact than the resection rates on the deterministic base-case ICER (resection rates decreased to sixth place).

Probabilistic sensitivity analysis results are shown on cost-effectiveness scatter plots (Fig. 3) and by cost-effectiveness acceptability curves (CEAC; Fig. 4). To test overall model uncertainty, results for the $£ 30,000$ and $£ 50,000$ WTP thresholds are shown. The scatter plots indicate that treatment with cetuximab plus FOLFIRI versus FOLFIRI alone is costlier but also more effective 
ITT

Base-case ICER $=£ 130,929$

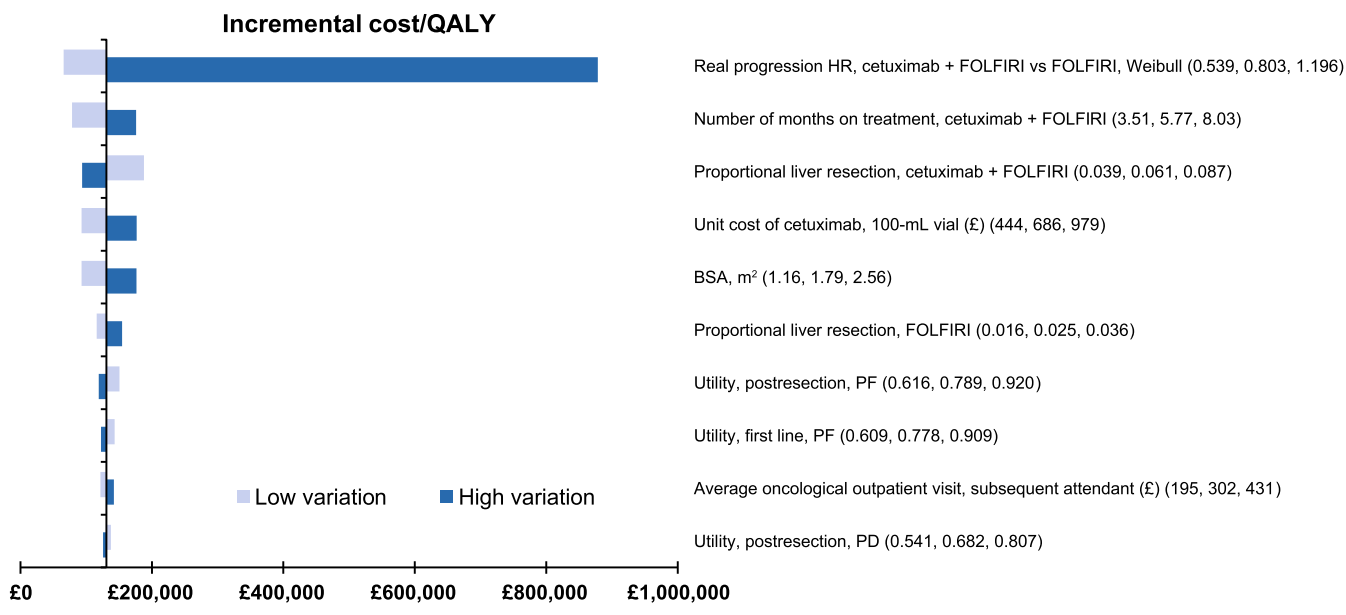

b

KRAS wt

Base-case ICER $=£ 72,053$

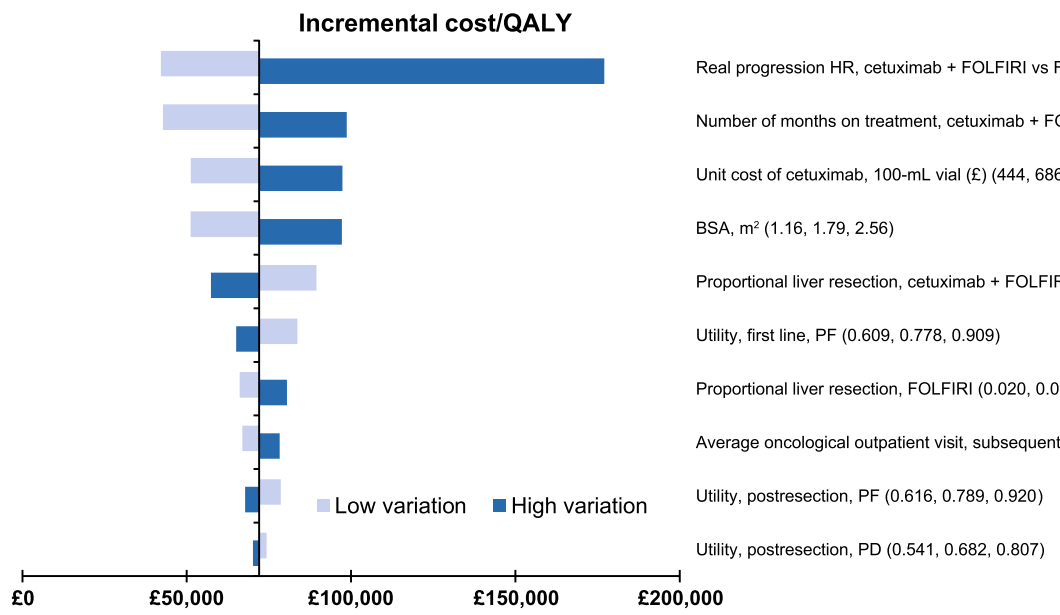

C

RAS wt
Base-case ICER $=£ 44,185$

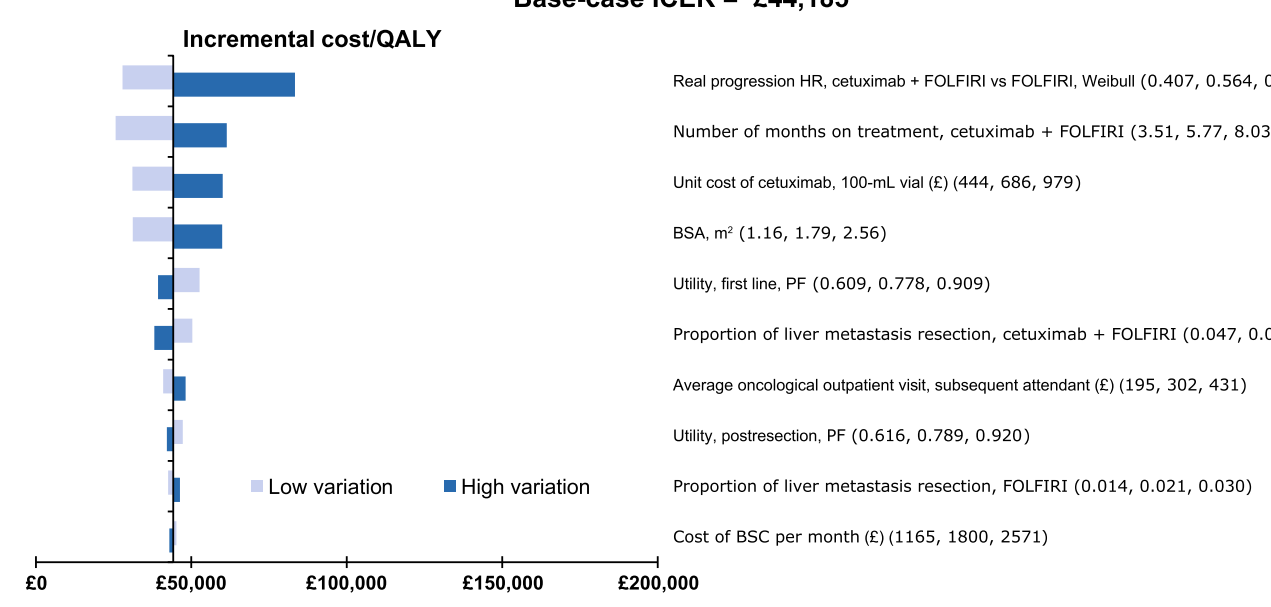

Real progression HR, cetuximab + FOLFIRI vs FOLFIRI, Weibull $(0.407,0.564,0.782)$

Number of months on treatment, cetuximab + FOLFIRI $(3.51,5.77,8.03)$

Unit cost of cetuximab, 100-mL vial (f) $(444,686,979)$

BSA, $m^{2}(1.16,1.79,2.56)$

(

Utility, postresection, PF $(0.616,0.789,0.920)$

Cost of BSC per month $(£)(1165,1800,2571)$

$£ 0 \quad £ 50,000 \quad £ 100,000 \quad £ 150,000 \quad £ 200,000$ 
4Fig. 2 One-way sensitivity analysis results. Results of the OWSA on the deterministic base-case ICER for the cetuximab-plus-FOLFIRI versus FOLFIRI-alone comparisons for the a ITT cohort, b KRAS wt cohort and $\mathbf{c}$ RAS wt cohort. Values provided for each entry represent the upper and lower limits generated by using the 0.025 percentile as a lower bound and the 0.975 percentile as an upper bound of the relevant variable. $B S A$ body surface area, $B S C$ best supportive care, FOLFIRI 5-fluorouracil and leucovorin plus irinotecan, $H R$ hazard ratio, ICER incremental cost-effectiveness ratio, ITT intent-to-treat, $K R A S$ Kirsten rat sarcoma viral oncogene homolog, OWSA one-way sensitivity analysis, $P D$ progressive disease, $P F$ progression-free, $Q A L Y$ quality-adjusted life-year, $R A S$ rat sarcoma oncogene

(Fig. 3). The least favourable distribution occurred in the ITT cohort (Fig. 3a), where some of the simulations fell into the northwest quartile, where cetuximab plus FOLFIRI would be considered costlier and less effective than FOLFIRI alone. ICER plots shift further east in the quartile as the cohorts move from ITT to KRAS wt (Fig. 3b) and RAS wt (Fig. 3c) based on increased efficacy of cetuximab in these patient groups and result in an increasing proportion of the ICERs below the WTP line. The clusters become more diffuse, indicating more uncertainty as numbers of patients in the sample are reduced. Using a $£ 50,000$ WTP threshold, the RAS wt cohort would still have the highest probability of being cost-effective compared with the other cohorts (Fig. 3). Results of CEACs indicate the probability that the treatment strategy of each cohort is cost-effective at different WTP threshold per QALY thresholds. Figure 4 shows that, at the $£ 30,000$ WTP threshold, cetuximab plus FOLFIRI in the ITT cohort has a $0 \%$ probability of being cost-effective, and, in the KRAS wt cohort, there is only a $5 \%$ chance that it is cost-effective. However, in the RAS wt cohort, at the $£ 30,000$ limit, there is a $15 \%$ probability that cetuximab plus FOLFIRI versus FOLFIRI alone in this group is cost-effective. The probability that the treatment strategy of each population is cost-effective at a WTP threshold of $£ 50,000$ is $<5 \%$ for the ITT population, $20 \%$ for the KRAS wt group and $63 \%$ for the RAS wt group.

\section{Discussion}

Research to date has shown that the majority of treatments prescribed for various disease conditions are effective in $<60 \%$ of treated patients (25-30\% in oncology), underscoring the potential (and need) for efficiency gains in healthcare delivery [37]. Stratified medicine is a new approach that may improve medical outcomes for the patient and the healthcare system by matching therapies to specific patient populations using clinical biomarkers and diagnostics. Stratified medicine can enhance patient care through the development and administration of safer and more effective drugs delivered with a greater chance of successful treatment. Additionally, more accurate targeting of patients with the most effective medication will decrease the burden on healthcare systems by more efficient healthcare delivery [37]. Despite the potential for stratified medicine to foster greater success in treatment outcomes, it is currently not widely used, due, in part, to scientific barriers, economic concerns and difficulties in securing coverage and adequate reimbursement. Many currently available tests do not have the sensitivity required to identify clinically meaningful differences between patient populations to facilitate effective stratification [37].

Healthcare interventions with demonstrated clinical efficacy and marketing authorisation may not ultimately be used in the originally intended population, as access to the healthcare system may be decreased due to a high ICER. However, a treatment that is not used cannot offer a benefit to the patient or the healthcare system. The aim of this study was to investigate this question through an economic evaluation of the three cohorts (ITT, KRAS wt and RAS wt) from the CRYSTAL study and determine if the stratification of cetuximab plus FOLFIRI versus FOLFIRI by biomarker status improves its cost-effectiveness. Based on our results, among patients with mCRC treated with antieGFR therapy, treatment of patients in the RAS wt cohort demonstrated the most cost-effectiveness of the three cohorts.

Our results are consistent with those of previous studies that support the notion that targeting patients with RAS wt tumours is more cost-effective than treating patients with KRAS wt tumours; however, our study is unique in that it compares three cetuximab populations at once. The FIRE-3 trial $[38,39]$ compared cetuximab plus FOLFIRI with bevacizumab plus FOLFIRI (not FOLFIRI alone) and compared only the KRAS wt versus the RAS wt populations. In that study, first-line treatment alone and not sequential use of the products likely drove the outcome [40], whereas the CRYSTAL data demonstrated a clear implication that cetuximab drives the improved clinical benefit and cost-effectiveness in targeting therapy. Additionally, Wen et al. [39] discussed the added costs of RAS screening versus KRAS screening and displayed results in quality-adjusted life-months. Kircher et al. [41] found that the increased societal cost of expanded RAS testing versus standard approved KRAS exon 2 testing was inconsequential compared with the savings achieved by not treating the $18 \%$ of patients who harbour additional RAS mutations (beyond exon 2) with anti-EGFR therapy. Another study revealed that additional savings may be obtained when testing for mutations in the BRAF gene is added to KRAS screening [42].

In this study, the economic analysis revealed that variation in incremental cost was driven by the changing proportion of patients in the cetuximab-plus-FOLFIRI or the 
Fig. 3 Probabilistic sensitivity analysis results on costeffectiveness scatter plots. To test overall model uncertainty, results for the $£ 30,000$ (beige dotted line) and $£ 50,000$ (green dotted line) WTP thresholds are shown. Point estimates, represented by dots beneath each dotted line, reflect simulations falling under each WTP threshold. Results are presented for the a ITT, b KRAS wt and c RAS wt cohorts. ITT intent-to-treat, KRAS Kirsten rat sarcoma viral oncogene homolog, $Q A L Y$ quality-adjusted life-year, $R A S$ rat sarcoma oncogene, $w t$ wild type, WTP willingness to pay
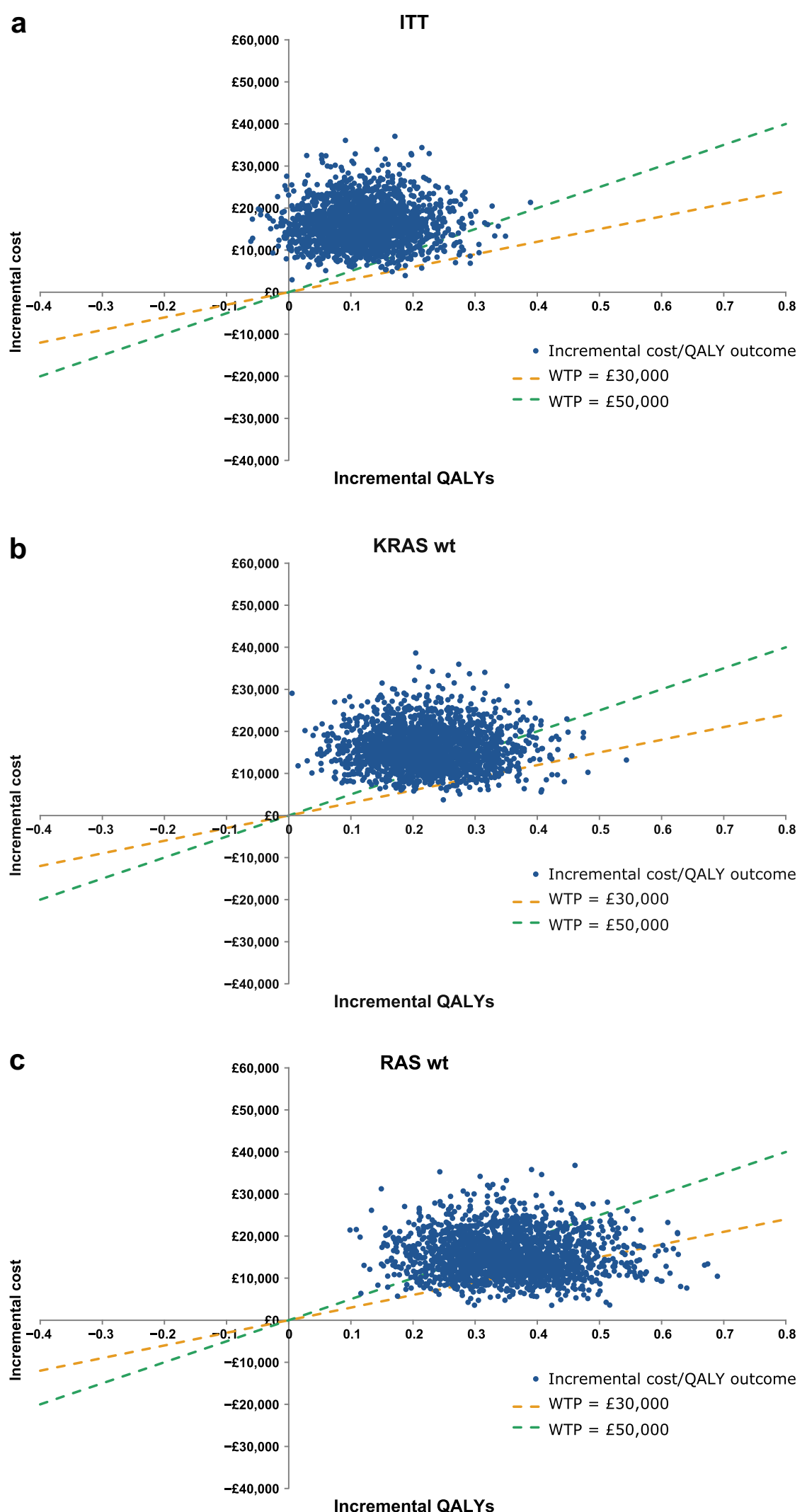


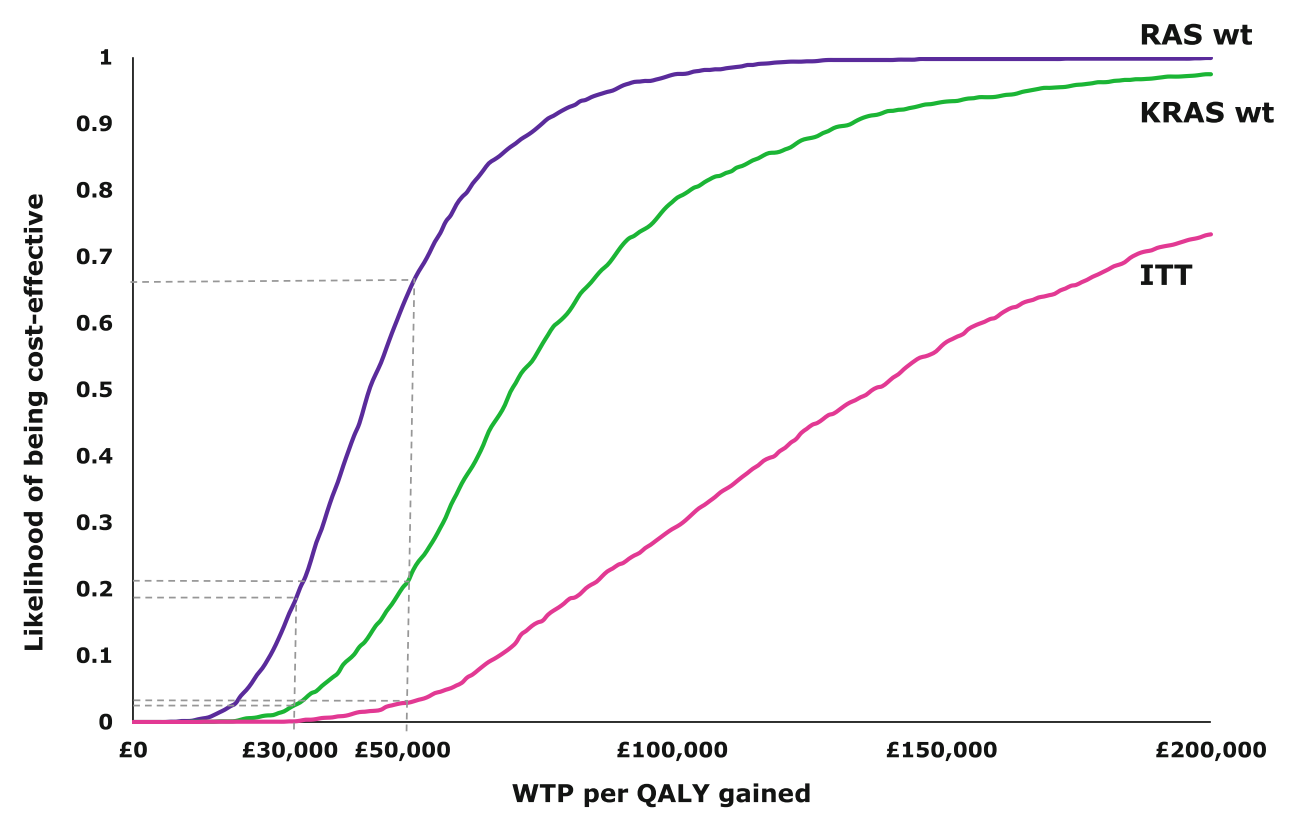

Fig. 4 Cost-effectiveness acceptability curves for the probabilistic sensitivity analysis. Dotted lines show probabilities for each cohort at the $£ 30,000$ and $£ 50,000$ WTP thresholds. Note, at the $£ 30,000$ WTP threshold, cetuximab plus FOLFIRI versus FOLFIRI alone had a $0 \%$ probability of being cost-effective for the ITT cohort (red line), $5 \%$ for the KRAS wt cohort (green line) and $15 \%$ for the RAS wt cohort (blue

FOLFIRI-alone arms who received curative surgery and no longer required second- or third-line chemotherapy. Even with the costs of biomarker testing considered, the RAS wt cohort had the lowest overall costs of treatment in the cetuximab arm and the lowest incremental cost of treatment of all the groups.

The OWSA model was particularly sensitive to the time spent in first-line progression. It could be determined from the OWSA that the variables around the hazard function in first-line progression (derived from the Weibull distribution) determine the greatest variation around the base-case ICER. This variation was particularly large in the ITT group, as the upper-bound HR crossed above 1.0. The HR of progression and the number of months on treatment were the variables that had the most impact on the model. In the tornado diagrams, acquisition costs associated with cetuximab are among the largest determinants of the ICERs; however, overall, the relative influence of variables on the ICER changes between the groups. Results of our PSA analysis support the OWSA findings. The shift in the ICER pointed toward greater benefit, and the increasing proportion below the WTP thresholds visually demonstrates the improving ICERs as the sample size decreases from the ITT and KRAS wt to RAS wt cohorts due to extended restriction of the population of interest based on biomarkers. In the ITT scatter plot, modelled observations are located in the northwest quadrant, representing a level of unfavourability in the ITT group. In these scenarios, line). At the $£ 50,000 \mathrm{WTP}$ threshold, the probability that the treatment strategy is cost-effective was $<5 \%$ for the ITT, $20 \%$ for the KRAS wt and $>60 \%$ for the RAS wt cohorts. FOLFIRI 5-fluorouracil and leucovorin plus irinotecan, ITT intent-to-treat, KRAS Kirsten rat sarcoma viral oncogene homolog, $Q A L Y$ quality-adjusted life-year, $R A S$ rat sarcoma oncogene, $w t$ wild type, $W T P$ willingness to pay

cetuximab treatment would not be beneficial in this small proportion of patients and may cause harm. Additionally, it should be noted that even targeting therapy by treating RAS wt patients still results in a relatively low cost-effectiveness probability of $63 \%$.

Considering the findings of our study, it should be noted that our results may have been limited by the fact that post hoc analysis or retrospective auditing of clinical studies is not generally considered the best source of evidence due to risks associated with bias. Additionally, a disease-modelling approach was used (assuming the PFS benefits of first-line cancer treatments translate directly into OS benefits), and in this evaluation parametric curves were fitted to the PFS data. In the case of the CRYSTAL study and subsequent analysis, the PFS results do transform into OS gains. However, for completion, repeating the analysis using the OS data would be the next logical step. This may have an impact on the final deterministic ICER results, but it should not alter the cost-effective trends between the cohorts and it is likely to result in a similar conclusion.

\section{Conclusions}

Targeting therapy to patients based on a genetic biomarker can notably decrease the ICER, which demonstrated that the increase in benefit to patients did outweigh the 
increased costs to the healthcare system. The base-case deterministic ICER results demonstrated that the ICER declined as the patient population was stratified for the RAS biomarker. The improved ICER corresponded with the increase in survival benefit from cetuximab seen for those patients with the tumour biomarker (KRAS or RAS). The RAS wt cohort had the lowest ICER; therefore, cetuximab plus FOLFIRI was the most cost-effective versus FOLFIRI alone in this subgroup at a WTP threshold of $£ 50,000$.

Acknowledgements The authors thank Philipp von Hohnhorst and colleagues at MAPI for technical assistance. We also thank colleagues at Merck for providing the data and manuscript development support. Portions of this study were presented in poster format at the International Society for Pharmacoeconomics and Outcomes Research 18th Annual European Congress on 9 November 2015 in Milan, Italy.

Author Contribution All of the authors were involved in the project design, the model development, data analysis and writing of this research article.

\section{Compliance with Ethical Standards}

Gerard Harty is an employee of Merck KGaA. James Jarrett is a former employee of MAPI, and was employed by this company during this research. This employer was compensated by a range of pharmaceutical, medical device and vaccine companies; the clients of this employer are considered confidential information. Mireia JofreBonet has been compensated for consulting services by AstraZeneca.

Funding This study was sponsored by Merck KGaA.

Open Access This article is distributed under the terms of the Creative Commons Attribution-NonCommercial 4.0 International License (http://creativecommons.org/licenses/by-nc/4.0/), which permits any noncommercial use, distribution, and reproduction in any medium, provided you give appropriate credit to the original author(s) and the source, provide a link to the Creative Commons license, and indicate if changes were made.

\section{References}

1. Jones RT, Felsenstein KM, Theodorescu D. Pharmacogenomics: biomarker-directed therapy for bladder cancer. Urol Clin North Am. 2016;43(1):77-86. https://doi.org/10.1016/j.ucl.2015.08. 007.

2. He J, Ahuja N. Personalized approaches to gastrointestinal cancers: importance of integrating genomic information to guide therapy. Surg Clin North Am. 2015;95(5):1081-94. https://doi. org/10.1016/j.suc.2015.05.002.

3. Carper MB, Claudio PP. Clinical potential of gene mutations in lung cancer. Clin Transl Med. 2015;4(1):33. https://doi.org/10. 1186/s40169-015-0074-1.

4. Lumachi F, Chiara GB, Foltran L, Basso SM. Proteomics as a guide for personalized adjuvant chemotherapy in patients with early breast cancer. Cancer Genom Proteom. 2015;12(6):385-90.

5. Shabaruddin FH, Fleeman ND, Payne K. Economic evaluations of personalized medicine: existing challenges and current developments. Pharmacogenom Pers Med. 2015;8:115-26. https://doi.org/10.2147/pgpm.s35063.

6. ABPI (2014) conference report, stratified medicine: discovery to patient-mind the gap. http://www.abpi.org.uk/our-work/library/ industry/Documents/strat_med_conference_report_2014.pdf. Accessed 8 Jan 2016.

7. Oosterhoff M, van der Maas ME, Steuten LM. A systematic review of health economic evaluations of diagnostic biomarkers. Appl Health Econ Health Policy. 2016;14(1):51-65. https://doi. org/10.1007/s40258-015-0198-x.

8. National Institute for Health and Care Excellence. Colorectal cancer: diagnosis and management. 2011. http://www.nice.org. uk/guidance/cg131. Accessed 8 Jan 2016.

9. Ciardiello F, Tortora G. A novel approach in the treatment of cancer: targeting the epidermal growth factor receptor. Clin Cancer Res. 2001;7(10):2958-70.

10. Ciardiello F, Tortora G. EGFR antagonists in cancer treatment. N Engl J Med. 2008;358(11):1160-74. https://doi.org/10.1056/ NEJMra0707704.

11. Cohen SJ, Cohen RB, Meropol NJ. Targeting signal transduction pathways in colorectal cancer-more than skin deep. J Clin Oncol. 2005;23(23):5374-85. https://doi.org/10.1200/jco.2005.02.194.

12. Hecht JR, Douillard JY, Schwartzberg L, Grothey A, Kopetz S, Rong A, et al. Extended RAS analysis for anti-epidermal growth factor therapy in patients with metastatic colorectal cancer. Cancer Treat Rev. 2015;41(8):653-9. https://doi.org/10.1016/j. ctrv.2015.05.008.

13. Bokemeyer C, Kohne CH, Ciardiello F, Lenz HJ, Heinemann V, Klinkhardt $\mathrm{U}$, et al. FOLFOX4 plus cetuximab treatment and RAS mutations in colorectal cancer. Eur $\mathrm{J}$ Cancer. 2015;51(10):1243-52. https://doi.org/10.1016/j.ejca.2015.04.007.

14. Van Cutsem E, Lenz HJ, Kohne CH, Heinemann V, Tejpar S, Melezinek I, et al. Fluorouracil, leucovorin, and irinotecan plus cetuximab treatment and RAS mutations in colorectal cancer. J Clin Oncol. 2015;33(7):692-700. https://doi.org/10.1200/jco. 2014.59.4812.

15. Bronte G, Silvestris N, Castiglia M, Galvano A, Passiglia F, Sortino G, et al. New findings on primary and acquired resistance to anti-EGFR therapy in metastatic colorectal cancer: do all roads lead to RAS? Oncotarget. 2015;6(28):24780-96. https://doi.org/ 10.18632/oncotarget.4959.

16. Inoue $M$, Takahashi $S$, Soeda $H$, Shimodaira $H$, Watanabe $M$, Miura K, et al. Gene-expression profiles correlate with the efficacy of anti-EGFR therapy and chemotherapy for colorectal cancer. Int J Clin Oncol. 2015;20(6):1147-55. https://doi.org/10. 1007/s10147-015-0841-4.

17. Van Cutsem E, Kohne CH, Hitre E, Zaluski J, Chang Chien CR, Makhson A, et al. Cetuximab and chemotherapy as initial treatment for metastatic colorectal cancer. N Engl J Med. 2009;360(14):1408-17. https://doi.org/10.1056/NEJMoa0805019.

18. Van Cutsem E, Kohne CH, Lang I, Folprecht G, Nowacki MP, Cascinu $\mathrm{S}$, et al. Cetuximab plus irinotecan, fluorouracil, and leucovorin as first-line treatment for metastatic colorectal cancer: updated analysis of overall survival according to tumor KRAS and BRAF mutation status. J Clin Oncol. 2011;29(15):2011-9. https://doi.org/10.1200/jco.2010.33.5091.

19. NCCN. NCCN Clinical Practice Guidelines in Oncology. Colon Cancer. V2.2016. http://www.nccn.org/professionals/physician_ gls/pdf/colon.pdf. Accessed 11 Jan 2016.

20. Van Cutsem E, Cervantes A, Nordlinger B, Arnold D. Metastatic colorectal cancer: ESMO Clinical Practice Guidelines for diagnosis, treatment and follow-up. Ann Oncol. 2014;25(Suppl 3):1-9. https://doi.org/10.1093/annonc/mdu260.

21. Scottish Medicines Consortium. Cetuximab, $100 \mathrm{mg} / 20 \mathrm{~mL}$ and $500 \mathrm{mg} / 100 \mathrm{~mL}$ solution for intravenous infusion (Erbitux). No. 543/09. https://www.scottishmedicines.org.uk/files/cetuximab_ 
Erbutux_FINAL_March_2009_Amended_26.06.09.pdf. Accessed 6 Apr 2016.

22. National Institute for Health and Care Excellence. Cetuximab for the first-line treatment of metastatic colorectal cancer. Issued: August 2009. https://www.nice.org.uk/guidance/ta176/resources/ cetuximab-for-the-firstline-treatment-of-metastatic-colorectalcancer-82598439035077. Accessed 6 Apr 2016.

23. Scottish Medicines Consortium. Cetuximab, $100 \mathrm{mg} / 20 \mathrm{~mL}$ and $500 \mathrm{mg} / 100 \mathrm{~mL}$ solution for intravenous infusion (Erbitux). No. 1012/14. http://www.scottishmedicines.org.uk/files/advice/ cetuximab_Erbitux_FINAL_Dec_2014_for_website.pdf. Accessed 6 Apr 2016.

24. All Wales Medicines Strategy Group (AWMSG). Final appraisal recommendation advice no: 4315-December 2015. Cetuximab $\left(\right.$ Erbitux $\left.^{\circledR}\right) 5 \mathrm{mg} / \mathrm{ml}$ solution for infusion. http://www.awmsg.org/ awmsgonline/grabber?resId=File\%2F2068. Accessed $6 \mathrm{Apr}$ 2016.

25. National Institute for Health and Care Excellence. Cetuximab and panitumumab for previously untreated metastatic colorectal cancer. Issued: March 2017. https://www.nice.org.uk/guidance/ GID-TAG470/documents/final-appraisal-determination-document. Accessed 7 Mar 2017.

26. Bokemeyer C, Bondarenko I, Hartmann JT, de Braud F, Schuch G, Zubel A, et al. Efficacy according to biomarker status of cetuximab plus FOLFOX-4 as first-line treatment for metastatic colorectal cancer: the OPUS study. Ann Oncol. 2011;22(7):1535-46. https://doi.org/10.1093/annonc/mdq632.

27. Yamaguchi K, Ando M, Ooki A, Beier F, Guenther S, von Hohnhorst P, et al. Quality of life analysis in patients with RAS wild-type metastatic colorectal cancer treated with first-line cetuximab plus chemotherapy. Clin Colorectal Cancer. 2016. https://doi.org/10.1016/j.clcc.2016.07.017.

28. Dias S, Sutton AJ, Welton NJ, Ades AE. NICE DSU technical support document 6: embedding evidence synthesis in probabilistic cost-effectiveness analysis: software choices. ScHARR, University of Sheffield: Decision Support Unit; 2012.

29. Hnoosh A, Harty GT, Sullivan L, Byrne B, von Honhorst P. Cost effectiveness of cetuximab in first line treatment of Ras wild-type metastatic colorectal cancer in the UK: a summary of economic analyses submitted to the National Institute for Health and Care Excellence (NICE). Value Health. 2015;18(7):A459. https://doi. org/10.1016/j.jval.2015.09.1182.

30. Samyshkin Y, Hertel N, Griebsch I. Cost-effectiveness of cetuximab and bevacizumab in the first-line treatment of metastatic colorectal cancer (mCRC) for patients with Kras wild-type tumours in the United Kingdom. Value Health. 2011;14(7):A446-7.

31. National Institute for Health and Care Excellence. Cetuximab and panitumumab for previously untreated metastatic colorectal cancer. Technology appraisal guidance [TA439]. 2017. https:// www.nice.org.uk/guidance/ta439. 18 Aug 2017.

32. Feiller N. Survival data analysis. Course booklet. Probability \& statistics, SoMaS. University of Sheffield. 2012. www.nickfieller. staff.shef.ac.uk/tampere12/survivalprint.pdf. Accessed 8 Jan 2016.

33. Adam R, Delvart V, Pascal G, Valeanu A, Castaing D, Azoulay $\mathrm{D}$, et al. Rescue surgery for unresectable colorectal liver metastases downstaged by chemotherapy: a model to predict long-term survival. Ann Surg. 2004;240(4):644-57 (discussion 57-8).

34. Van Krieken JHJM, Rouleau E, Ligtenberg MJL, Normanno N, Patterson SD, Jung A. RAS testing in metastatic colorectal cancer: advances in Europe. Virchows Arch. 2016;468:383-96. https://doi.org/10.1007/s00428-015-1876-7.

35. Briggs A, Claxton K, Sculpher M. Decision modelling for health economic evaluation (handbooks in health economic evaluation). Oxford: Oxford University Press; 2011.

36. National Institute for Health and Care Excellence. Value based assessment of health technologies. National Institute for Health and Care Excellence Centre for Health technology evaluation consultation paper. 2013. https://www.nice.org.uk/Media/Default/ About/what-we-do/NICE-guidance/NICE-technology-appraisals/ VBA-TA-Methods-Guide-for-Consultation.pdf. Accessed 22 Nov 2016.

37. Fugel HJ, Nuijten M, Postma M. Stratified medicine and reimbursement issues. Front Pharmacol. 2012;3:181. https://doi.org/ 10.3389/fphar.2012.00181.

38. Shankaran V, Ortendahl JD, Purdum AG, Bolinder B, Anene AM, Sun GH, et al. Cost-effectiveness of cetuximab as first-line treatment for metastatic colorectal cancer in the United States. Am J Clin Oncol. 2018;41(1):65-72.

39. Wen F, Yang Y, Zhang P, Zhang J, Zhou J, Tang R, et al. Costeffectiveness of RAS screening before monoclonal antibodies therapy in metastatic colorectal cancer based on FIRE3 study. Cancer Biol Ther. 2015;16(11)1577-84.

40. Modest DP, Stintzing S, von Weikersthal LF, Decker T, Kiani A, Vehling-Kaiser U, et al. Impact of subsequent therapies on outcome of the FIRE-3/AIO KRK0306 trial: first-line therapy with FOLFIRI plus cetuximab or bevacizumab in patients with KRAS wild-type tumors in metastatic colorectal cancer. J Clin Oncol. 2015;33(32):3718-26. https://doi.org/10.1200/jco.2015.61.2887.

41. Kircher SM, Mohindra N, Nimeiri H. Cost estimates and economic implications of expanded RAS testing in metastatic colorectal cancer. Oncologist. 2015;20(1):14-8. https://doi.org/10. 1634/theoncologist.2014-0252.

42. Behl AS, Goddard KA, Flottemesch TJ, Veenstra D, Meenan RT, Lin JS, et al. Cost-effectiveness analysis of screening for KRAS and BRAF mutations in metastatic colorectal cancer. J Natl Cancer Inst. 2012;104(23):1785-95. https://doi.org/10.1093/jnci/djs433. 